\title{
Use of Impella CP Device in Off-pump Coronary Artery Bypass Graft Surgery
}

\author{
Vandan Upadhyaya ${ }^{1}$, Stuart Campbell ${ }^{1}$, Ishan Patel $^{1}$, Steven Douedi ${ }^{1}$, Kourosh Asgarian ${ }^{1}$, \\ and Matthew Saybolt ${ }^{1}$ \\ ${ }^{1}$ Jersey Shore University Medical Center
}

June 1, 2020

\begin{abstract}
Introduction: Off-pump coronary artery bypass grafting (OPCABG) may be performed on patients with high surgical risk who are poor candidates for traditional mechanical circulatory support. Hemodynamic support with micro-axial mechanical circulatory devices has been performed with limited but promising results. Case Presentation: We report a case of 66-yearold male with multiple comorbidities and low cardiac output undergoing OPCABG. Impella CP device was deployed for "inpump" support during surgical coronary revascularization resulting in intraoperative stability and uncomplicated post-operative recovery. Discussion: Previous reports have described use of the Impella Recover LP 5.0 device for use during OPCABG. We describe the successful and safe perioperative use of the Impella CP device. Despite lower flow rates, adequate support was achieved and the transfemoral cannulation and smaller outer diameter than the Impella 5.0 device may decrease the risk of complications and expedite recovery. Conclusion: Further research will be necessary to determine the optimal perioperative hemodynamic support strategy to offer hemodynamically unstable, high and prohibitive risk patients.
\end{abstract}

\section{Introduction}

Off-pump coronary artery bypass graft (OPCABG), also known as "beating heart surgery", refers to coronary artery bypass grafting performed without cardiac arrest or cardiopulmonary bypass hemodynamic [1,2]. The technique is most commonly employed in patients with isolated proximal left anterior descending coronary artery (LAD) disease without or without associated proximal right coronary disease [2]. The procedure requires the expertise of a surgeon having undergone additional training in the performance of OPCABG. While it can be performed in individuals with low predicted risk of surgical morbidity or mortality, it is a technique that is appealing to offer in those at high risk from such comorbidities as hostile aorta, severe myocardial dysfunction, or underlying renal or pulmonary disease [3-5]. Meta-analysis and trials have shown no difference between on-pump CABG and OPCABG with respect to all-cause mortality but did find a $28 \%$ decrease in risk of stroke with the use of OPCABG $[6,15]$.

While high-risk patients are those that may derive the greatest benefit from OPCABG, they are the most vulnerable to intra-operative decompensation without circulatory support. Currently no percutaneous device is approved for mechanical circulatory support during OPCABG procedure. Use of micro-axial mechanical circulatory devices during OPCABG may prove to be a promising alternative. Currently Impella device (Abiomed, Danvers, MA, USA) considers manipulations of the heart that would be used during cardiothoracic surgery to be a contraindication to the use of the device $[7,8]$. Despite the concern, select cases have been describing the use of Impella Recover LP 5.0 during OPCABG $[9,10]$. Impella 5.0 delivers upwards of 5.0 $\mathrm{L} / \mathrm{min}$ support at the consequence of a 21 French inner diameter sheath through a surgically cannulated axillary arteriotomy. 
We describe the use of the smaller caliber Impella CP (Abiomed, Danvers, MA, USA) with maximum output of $3.7 \mathrm{~L} / \mathrm{min}$, in a case of OPCABG with favorable intraoperative and post-operative results.

\section{Case Presentation}

A 66-year-old male with past medical history of hypertension, hyperlipidemia, coronary artery disease, systolic cardiomyopathy with an ejection fraction of $25 \%$ and a primary prevention biventricular ICD presented to outside facility with unstable angina and aborted sudden cardiac death. He had been treated with several rounds of anti-tachycardia pacing and at least 6 internal defibrillations. On arrival he was the midst of incessant episodes of pace terminated ventricular tachycardia. He was started on intravenous amiodarone and transferred to the cardiac care unit at our hospital.

Peak troponin I level prior to arrival was 7.48 and under the circumstances he was taken for emergent coronary angiography. We identified $>90 \%$ calcified stenosis of proximal left anterior descending coronary artery, proximal right coronary artery and ramus intermedius respectively with moderate distal left main tapering. We also identified high grade mid left anterior descending bifurcation stenosis involving the origin of the first diagonal (medina 0,1,1) (Figure 1). The anatomic Syntax score was 41 (high). His angina and arrythmias became more quiescent with medical therapy and he recovered in the coronary care unit where the heart team was convened. On further work up he was found to have left ventricular ejection fraction of $25-30 \%$, moderate left ventricular dilation, stage 3 chronic renal failure, and a heavily calcified ascending aorta. Given his age of only 67 years old, left ventricular systolic dysfunction, high anatomic Syntax score and risk of acute renal failure with repeated contrast dye exposures our team opted to offer OPCABG.

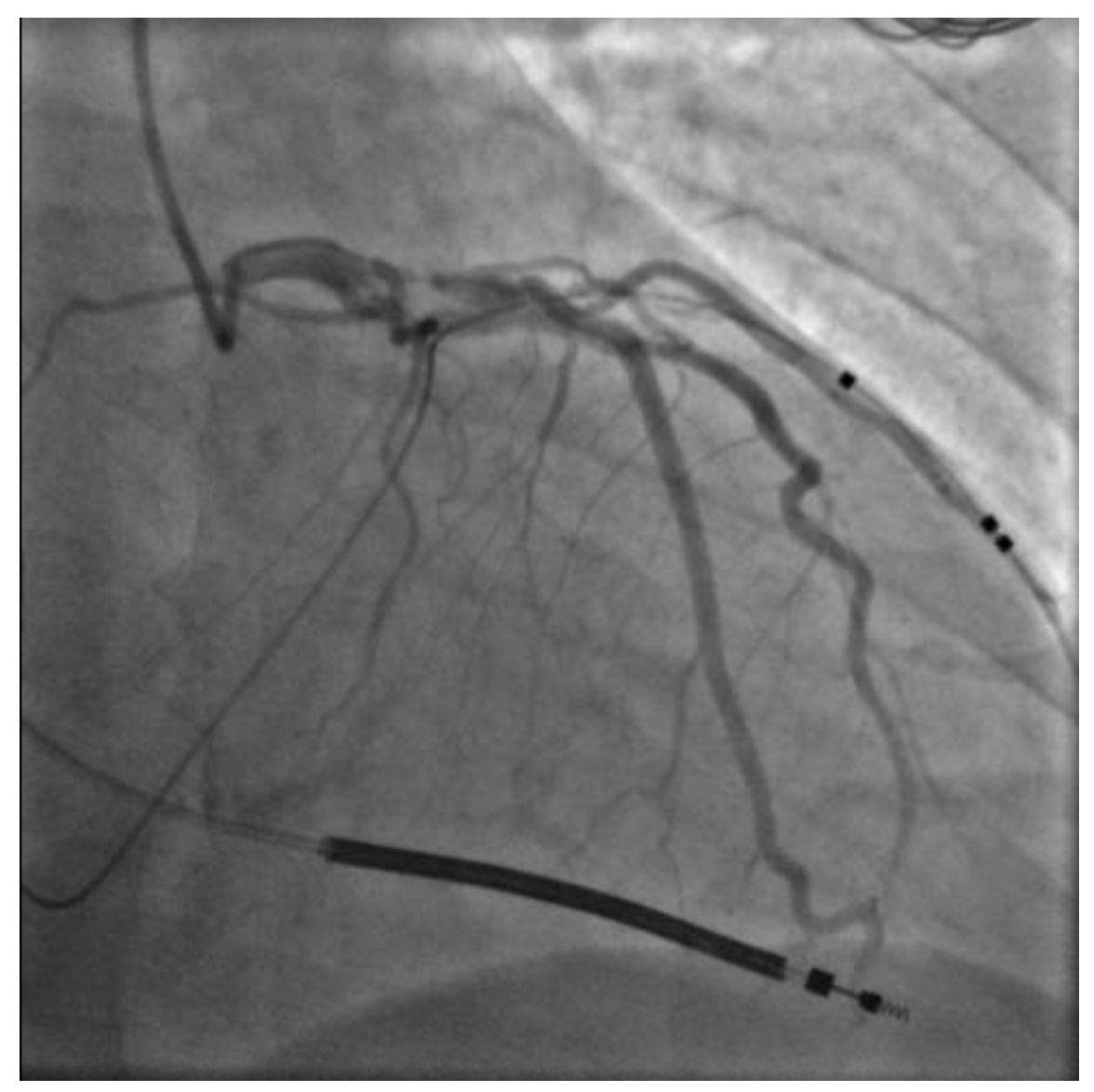

Figure 1: Right anterior oblique with cranial view (1A) and caudal view (1B) of left coronary system. 1C: 
Left anterior oblique with cranial view of right coronary system.

He was brought back to the cardiac catheterization lab pre-operatively and an Impella CP Device (Abiomed, Danvers, MA) inserted via the right common femoral artery in the usual fashion. The Impella CP remained in was used during the CABG operation as "in-pump" support and a standard OPCABG was performed utilizing a pedicle left internal mammary artery (LIMA), and endoscopically harvested reverse great saphenous vein (SVG). The LIMA was dissected out, and the distal targets were identified. The heart was elevated with moist lap pads taking care not to disrupt the Impella CP device. The target vessels were stabilized with an Octupus AS tissue stabilizer (Medtronic) and the distal anastomosis were completed. Three revascularization targets were achieved before hypotension began to progress (LIMA to left anterior descending; SVG to right coronary artery; SVG to ramus intermedius). The diagonal artery was left for medical therapy. The two proximal aortic anastomoses were created using HEARTSTRING III proximal seal system (Getinge). The Impella $\mathrm{CP}$ remained in place post operatively and the patient was taken back to the cardiac surgery intensive care unit. The Impella CP device was subsequently weaned and removed on post-operative day three in the cardiac catheterization lab and hemostasis obtained by tying down of Perclose Proglide (Abbott) sutures deployed at the time of the index procedure in the "preclose" technique. Completion ileo-femoral angiography acquired via the left radial artery demonstrated no vascular complication. The patient fared well and was transferred out of the intensive care using on post-operative day 5 where the remainder of his hospital stay was uneventful and was discharged on post-operative day 7 .

\section{Discussion}

Contradictory evidence exists in comparison of complications and all-cause mortality in trials involving off-pump CABG and conventional on-pump CABG. Meta-analysis performed have shown an increase in all-cause long-term mortality in patients with off-pump CABG compared to the traditional modality [11]. A 2014 meta-analysis of more than 20 studies found statistically significant $7 \%$ increase in long-term all-cause mortality with off-pump relative to on-pump CABG [11]. While in 2016, meta-analysis of 100 studies found no difference between the two techniques when comparing myocardial infarctions and all cause mortality [15]. A 2018 meta-analysis composed of more than 8000 patients reported higher mortality in OPCABG compared to on-pump CABG after 4 years or longer post procedure [16]. The population based evidence aside, each patient's clinical circumstances warrant a personalized approach.

There exist alternative strategies to improve outcomes and reduce peri-operative complications in off-pump coronary artery bypass grafting. Minimally invasive strategies employed during off-pump CABG, like minimally invasive direct CABG, have benefits that include faster recovery and improved results [12]. Percutaneous mechanical circulatory devices used during off-pump CABG may provide the additional circulatory support necessary during the necessary intra-operative cardiac manipulations and anesthesia that decrease cardiac output. Use of Impella devices have been previously described [13, 14]. Multiple cases have showed the utilization of Impella 5.0 device during off-pump- CABG $[13,14]$. The Impella 5.0 device utilizes a 21 French pump motor with peak flow up to $5.0 \mathrm{~L} / \mathrm{min}$.

The device used on our patient provides up to $3.5 \mathrm{~L} / \mathrm{min}$ of circulatory support. More important the Impella $\mathrm{CP}$ requires only only the insertion of additional peripheral vascular access via transfemoral approach with a lower profile system (Impella CP sheath outer diameter 17F, $\sim 4.7 \mathrm{~mm}$ ) when compared to the larger profile and more invasive Impella Recover LP 5.0 system.

Novel use of Impella $\mathrm{CP}$ with a peak flow rate of $3.5 \mathrm{~L} / \mathrm{min}$ in our case provided appropriate circulatory support for optimal recovery. Using the smaller motor size (catheter max outer diameter 14F) along with decreased power requirement may lead to decreased complications resulting from the use of Impella devices. Utility of smaller sized pump may have decreased the incidence of valve dysfunction, thromboembolic complications thrombosis, ventricular perforation, bleeding and vascular complications [8]. As this was an off-label use of the Impella device, caution should be advised prior to repeating our approach and further research is necessary to advance this burgeoning multi-disciplinary field.

\section{Conclusion}


We report a novel strategy utilizing an Impella CP for perioperative hemodynamic support in compromised patient undergoing high risk off-pump CABG with no complication and rapid recovery. The use of Impella CP device for patients undergoing off-pump CABG may improve outcomes and further studies should be performed to assess short- and long-term results in patients undergoing coronary revascularization through surgical means.

\section{References}

1. Khan N. E., De Souza A., Mister R., et al. A Randomized Comparison of Off-Pump and On-Pump Multivessel Coronary-Artery Bypass Surgery. The New England Journal of Medicine. 2004;350(1):21-28. doi: 10.1056/NEJMoa031282

2. Arom KV, Flavin TF, Emery RW, Kshettry VR, Janey PA, Petersen RJ. Safety and efficacy of off-pump coronary artery bypass grafting. Ann Thorac Surg. 2000;69:704-10.

3. Shroyer AL, et al. On-pump versus off-pump coronary-artery bypass surgery. N Engl J Med. 2009;361(19):1827-37. doi: 10.1056/NEJMoa0902905

4. Sharony R. Off-Pump Coronary Artery Bypass Grafting Reduces Mortality and Stroke in Patients With Atheromatous Aortas: A Case Control Study. Circulation. 2003;108(90101):15II—20

5. Ascione R, Nason G, Al-Ruzzeh S, et al. Coronary revascularization with or without cardiopulmonary bypass in patients with preoperative nondialysisdependent renal insufficiency. Ann Thorac Surg 2001;72:2020-5.

6. Sedrakyan A, Wu AW, Parashar A, Bass EB, Treasure T. Off-pump surgery is associated with reduced occurrence of stroke and other morbidity as compared with traditional coronary artery bypass grafting: a metaanalysis of systematically reviewed trials. Stroke. 2006;37(11):2759-69. 10.1161/01.STR.0000245081.52877.

7. Elhussein TA, Hutchison SJ. Acute mitral regurgitation: unforeseen new complica-tion of the Impella LP 5.0 ventricular assist device and review of literature. HeartLung Circ 2014;23:e100-4.

8. Ranc S, Sibellas F, Green L. Acute intraventricular thrombosis of an Impella LP 5.0device in an STelevated myocardial infarction complicated by cardiogenic shock.J Invasive Cardiol 2013;25:E1-3.

9. Akay MH, Frazier OH. Impella Recover 5.0 assisted coronary artery bypass grafting.J Card Surg 2010;25:606-7.

10. Yildiz CE, Sayin M, Yerebakan H, Kucukaksu S. First Turkish experiences of assistedbeating-heart coronary artery bypass graft with the Impella Microaxial Ventricu-lar Assist Device. Heart Surg Forum 2010;13:E60-2.

11. Takagi H, Umemoto T. All-Literature Investigation of Cardiovascular Evidence G. Worse longterm survival after off-pump than on-pump coronary artery bypass grafting. J Thorac Cardiovasc Surg. 2014;148(5):1820-9.

12. Holzhey DM, Jacobs S, Walther T, et al. Cumulative sum failure analysis for eight surgeons performing minimally invasive direct coronary artery bypass. J Thorac Cardiovasc Surg 2007; 134:663.

13. Pepino P, Coronella G, et al. Successful use of the Impella Recover LP 5.0 device for circulatory support during coronary artery bypass grafting. Int J Surg Case Rep. 2014;5(11):803-5.

14. Mehmet H., Akay MD, et al. Impella Recover 5.0 Assisted Coronary Artery Bypass Grafting. J Card Surg. 2010 Sep;25(5):606-7

15. Off-pump coronary artery bypass grafting improves short-term outcomes in high-risk patients compared with on-pump coronary artery bypass grafting: meta-analysis. Kowalewski M, Pawliszak W, Malvindi PG, et al. J Thorac Cardiovasc Surg. 2016;151:60-77.

16. Smart NA, Dieberg G, King N. Long-Term Outcomes of On- Versus Off-Pump Coronary Artery Bypass Grafting. J Am Coll Cardiol 2018; 71:983. 


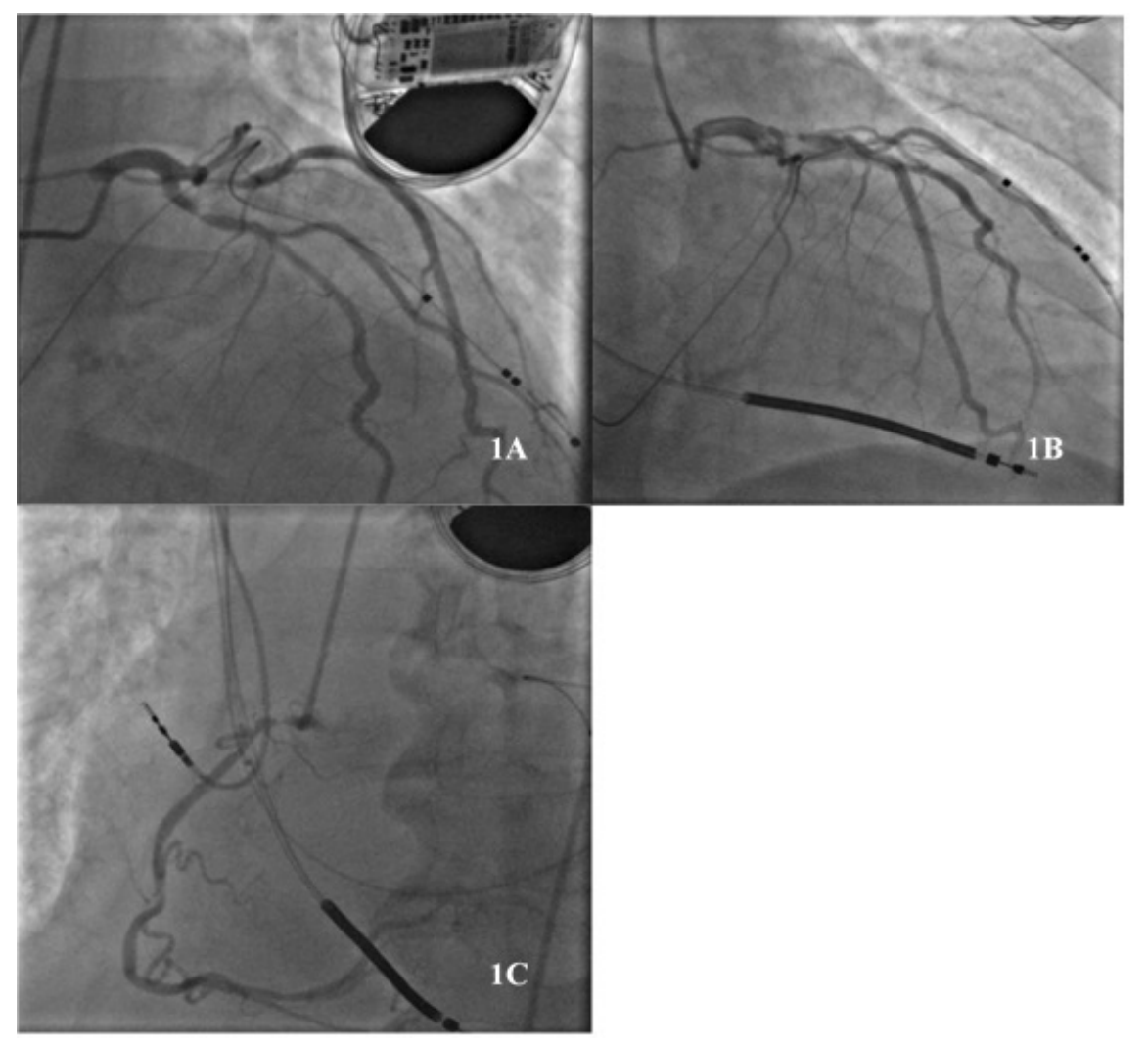

